\title{
Dietary Diversity among Indian adolescents and young adults: Evidence from UDAYA study
}

Anjali Bansal ( $\square$ anjali.bansal35@gmail.com )

International Institution for Population Sciences

Pooja Arora

International Institution for Population Sciences

Radhika Sharma

International Institution for Population Sciences

Archa Misra

Institute of Economic Growth

\section{Research Article}

Keywords: Dietary Diversity, India, Adolescents, Nutrition

Posted Date: December 8th, 2021

DOI: https://doi.org/10.21203/rs.3.rs-242191/v2

License: (c) (1) This work is licensed under a Creative Commons Attribution 4.0 International License.

Read Full License 
1 Dietary Diversity among Indian adolescents and young adults: Evidence from UDAYA study

2 Abstract

3 Objective: This work studied minimum dietary diversity (MDD) and explored its linkages with

4 background characteristics like household consumption behavior, presence of grandparents in the

5 household, number of siblings, involvement in paid work, etc.

6 Design: For bivariate analysis and sex differentials, chi-square test was done to study the 7 association between MDD and different covariates. Logistic regression analysis was performed to 8 identify determinants of MDD.

9 Setting: Data was collected from two majorly populous and backward states of India, namely 10 Bihar and Uttar Pradesh (UP).

11 Participants: Follow-up survey of the UDAYA study (2018-19) was conducted among 12 adolescents and young adults aged 12-23 years old.

13 Results: We found the prevalence of MDD to be 59\% among males and 56\% among females.

14 Bihar performed better overall with higher MDD and lesser gender inequality. Wealth Index and 15 caste were observed to be significantly associated with MDD. Food Consumption Score (FCS) of 16 the household and media exposure were significantly impacted the MDD.

17 Conclusions: Improving dietary practices at a younger age eventually results in improved 18 nutritional status and overall development of an individual. It can serve as the key to prevent any 19 nutritional deficiencies and diseases linked with it at later ages. The government should focus more 20 on imparting healthy practices related to diet among both adolescents and their families. Action is 21 required to refine the current schemes present for improvement in household food consumption, 22 more so for the poorer population. Programs are needed that work on reducing gender inequalities, 23 especially in the state of UP.

24 Keywords: Dietary Diversity; India; Adolescents; Nutrition

\section{List of Abbreviations}

26 FCS- Food consumption score

27 MDD- Minimum dietary diversity 
28 UDAYA - Understanding the lives of adolescents and young adults

29 WHO - World Health Organization

$30 \mathrm{SC} / \mathrm{ST}$ - Schedule Caste/ Schedule Tribe

31 OBC- Other Backward Caste

32 ICDS- Integrated Child Development Services

33 FAO- Food and Agriculture Organization

\section{$34 \quad$ Introduction}

35 India's 253 million adolescent population (highest in the world) presents an unprecedented 36 opportunity as well as a challenge (1). Every fifth person in India is an adolescent (i.e., 10-19 years 37 of age). They can be divided into two categories - the younger (10-14 years) and the older (15-19 38 years), based on their behavioral attitudes and needs (2). Adolescence is a complex transitional 39 phase from childhood to adulthood, in which they undergo various rapid changes from physical 40 appearances to changes in their food habits $(3,4)$. The adolescence stage is considered the second 41 window of opportunity to improve nutritional status and ensure healthy life expectancy. Ample 42 evidence on dietary diversity shows a significant association with nutritional indicators (5). An 43 adequate diversified diet is vital for optimal health and the development of physical and cognitive 44 health. Adolescence malnutrition (overweight/underweight) is associated with early onset of Non45 Communicable Diseases and other morbidities that add on to Years Lived with Disability (YLD) 46 (6). Comprehensive national nutrition survey (CNNS) findings have highlighted the poor 47 nutritional status of adolescents. It measured BMI among adolescents and found $26.3 \%$ of 48 adolescent boys, $14.2 \%$ of adolescent girls to be moderately or severely thin. The survey has also 49 collected data on micronutrient deficiencies across all ages, from children aged five years to 50 adolescents. It found that one-fourth of adolescents were deficient in vitamin D were deficient in 51 vitamin B12 and folate. In contrast, one-third of adolescents have vitamin A deficiency (7).

52 Micronutrient deficiency during early childhood can often transverse into adolescence with a long53 term effect on health, cognition, education, and productivity (8). Dietary diversity prevents the 54 deficiency of micronutrients, and hence the onset of deficiency diseases and other related health 55 issues. Also, it is strongly associated with various factors such as food insecurity, socio-economic 
56 condition, educational status, knowledge of nutrition, taste preference, and cultural acceptability 57 (9).

58 Some research shows no difference in the intra-household allocation of food in the case of pre59 school and primary school-age children (10-13), but that it begins after a certain age, and the 60 difference persists. Gender-based disparity in intra-household diet consumption exists through all 61 life stages, but the gap widens markedly at 15 years old. Also, some studies have revealed that 62 gender bias influences dietary diversity too. A study found a pro-boy bias in dietary diversity only 63 among the children aged up to 24 months born to illiterate mothers (14). Poor dietary diversity 64 among female adolescents could have intergenerational consequences as well (15). A study found 65 children of teen mothers to be five percentage points more likely to be stunted than children of 66 adult mothers (16). Teen pregnancies persist as age-old traditions such as child marriage are still 67 practiced in some parts of India (1.5 million child marriages every year) (17). The 4th round of 68 NFHS report supports this evidence, as $32 \%$ of women who had given birth at that time did it 69 before reaching 18 years of age (16). The dietary diversity of these young mothers if poor, would 70 lead to the next generation being undernourished as well.

71 The government has launched various policies and intervention programs to create awareness 72 about the importance of dietary diversity but failed to monitor it, especially among adolescent 73 girls. Therefore, our study is an attempt to understand the dietary pattern and diversity and its 74 related factors among adolescents and young adults. The findings of our study could help plan 75 interventions to improve their dietary pattern, nutrition status, and the overall well-being of the 76 adolescents.

77 In our study, we have used the UDAYA longitudinal study design, which provides a unique 78 opportunity to determine the role of dietary patterns. This rich dataset can help analyze the 79 changing intentions and attitudes in terms of dietary habits. In this study, we tried to examine the 80 role of food consumption behavior of the household to the dietary diversity among adolescents 81 and young adults in UP and Bihar. 


\section{Methods}

83

84

85

86

87

88

89

90

91

92

93

94

95

96

97

98

99

100

101

102

103

104

105

106

107

108

109

110

\section{Data Source}

The UDAYA (Understanding the lives of adolescents and young adults) dataset has been used in the study conducted by the Population Council, New Delhi. UDAYA is a longitudinal study done in Uttar Pradesh and Bihar following a cohort of adolescents aged 10-19 years. These two northIndian states comprise $28 \%$ of the adolescent population in the country, given they are large, highly populated, predominantly rural, high poverty states (18).

The study used both cross-sectional and longitudinal designs for sampling at the point of wave 1, and a multi-stage systematic sampling design was employed. UDAYA was designed to provide estimates at two-time points for the state as a whole along with for the urban and rural areas of the state for each of the five categories of respondents, namely younger boys in ages 10-14, older boys in ages 15-19, younger girls in ages 10-14, unmarried older girls in ages 15-19, and married older girls in ages 15-19. A total of 150 primary sampling units (PSUs), 75 for rural and 75 for urban respondents, were sampled in each state using the Probability proportional to size (PPS) technique. PSUs list was stratified using four variables, namely, region, village/ward size, the proportion of the population belonging to scheduled castes and scheduled tribes, and female literacy. The household sample in rural areas was selected in three stages, while in urban areas, in four stages.

Data collection for Wave 1 was done in 2015-16, and after three years, data was collected for wave 2 data in 2018-19. This paper analyses dietary intake in the past 24 hours, and this information was collected only at wave 2 . Hence, for the current study, a cross-sectional sample of only wave 2 is used, consisting of information of 12-23 years old adolescents and young adults. For a fair comparison between boys and girls, a sample of married females was dropped as information for its counterpart, i.e., married males are not collected in the survey. The final sample consisted of 4221 male and 5987 unmarried female adolescents. Sampling weights were used as mentioned in the UDAYA guidelines.

The outcome variable of the study, i.e., minimum dietary diversity, was defined as the intake of food belonging to at least 5 or more food groups in the past 24 hours, as defined by FAO and FHI 360, 2016 (19). For the computation of the standard measure of Minimum dietary diversity (MDDW) for women, FAO defines 10 diverse types of food groups as Grains, white roots, and tubers, 
111 and plantains; Pulses (beans, peas, and lentils); Nuts and seeds; Dairy; Meat, poultry and fish;

112 Eggs; Dark green leafy vegetables; Other vitamin A-rich fruits and vegetables; Other vegetables 113 and; Other fruits. Out of these 10, UDAYA asked for information on only the first seven food 114 groups in its survey. For the last three food groups, questions were asked on the overall intake of 115 "fruits" or "vegetables" without emphasizing whether they were vitamin-A rich or not. To adjust 116 for this data limitation, a weightage of 1.5 was given instead of 1 to the two proxy food groups, 117 namely "Fruits" and "Vegetables", as they may or may not be rich in vitamin-A. This way, we got 118 the total dietary diversity score to fall in the range of 0 to 10 as it generally should, where consumption of food from five or more food groups was defined as the proxy measure of minimum dietary diversity for our study population.

121 Independent variables consisted of socio-demographic characteristics like age of the adolescent, 122 sex, completed years of education, mother's completed years of education, whether doing paid 123 work in the past one year and caste. Family arrangement was also studied using information on 124 number of siblings and presence of any grandparent in the household. Other determining factors 125 included media exposure, food consumption behavior and wealth index of the household, place of 126 residence, and state. An additive index of Media Exposure is created comprising eight factors 127 coded in a binary manner ( $1=$ Yes/High, $0=\mathrm{No} / \mathrm{Low})$. Eight factors include information on owning mobile; owning of Laptop; usage of social media in the last three years; frequent (high) or infrequent (low) indulgence in activities like watching television; reading of newspaper; listening of radio; watching movies; usage of internet. Here, frequent use refers to the responses "almost every day" and "at least once a week", whereas infrequent usage refers to "at least once a month", "rarely" and "not at all". The final added score ranges from 0 to 8 , which is then categorised in to three terciles as Low (0-3), Medium (4-5) and High (6-8).

Food consumption of the household is reflected using a proxy index given some data constraints.

136 The Standard Food Consumption Score (FCS) is an index developed by the World Food 137 Programme (WFP) in 1996 to represent household caloric availability (20). The FCS combines 138 household-level data on the diversity (quality) and frequency (quantity) of food groups consumed 139 over the previous seven days. It is then weighted according to the relative nutritional value of the 140 consumed food groups. For instance, food groups containing nutritionally dense foods, such as 141 animal products, are given greater weight than those containing less nutritionally dense foods, such 
142 as tubers. Broad food groups and associated FCS weights are: main staples — weighted at 2, 143 pulses — weighted at 3, vegetables — weighted at 1, fruit — weighted at 1, meat and fish — weighted 144 at 4 , milk — weighted at 4 , sugar — weighted at 0.5 , and oil —weighted at 0.5. Condiments can also 145 be captured but are weighted at 0 . Consumption frequencies are computed by adding the number 146 of days a food item is eaten in a week. Then they are added and rounded off to a maximum limit 147 of 7 per food group. An additive score combining the weighted consumption frequencies of each 148 food group results in a total score ranging from 0 to 112.

But in the UDAYA study, we get a data restriction. Instead of following the standard measure of 7-days recall, a 30-day recall is used, and information is collected on six responses, namely, whether a food item was eaten daily, once in a week, 2-3 times a week, once in two weeks, once in four weeks or never. For finding the number of days per week a food item was consumed, these responses were weighted with $7,1,2.5,0.5,0.25$, and 0 number of days, respectively. To represent the food consumption status of a household as poor, borderline or acceptable, cut-offs defined by WFP are $0-21 ; 21.5-35$, and $>35$. But these cut-offs are often criticized in literature. They are termed as subjective because assigning cut-off points to a continuous quantitative measure is usually a matter of analytical judgment about the extent to which such categorical cut-offs are universally applicable (Maxwell, 2013). Hence, instead of using these cut-offs, we have simply divided the score into three terciles as Low (0-70), Medium (70-85), High (85-112).

\section{Statistical Analysis}

162 For analysis, cross-tabulation and chi-square test were used to test independence of outcome 163 variable with various factors as mentioned above. Binary logistic regression was used to identify 164 determinants of minimum dietary diversity $(\mathrm{Yes}=1, \mathrm{No}=0)$ of male and female adolescents. 165 Adjusted odds ratios were computed for Uttar Pradesh and Bihar separately, as well as for the 166 combined sample.

\section{Findings}

168 Table 1 represents consumption of items from different food groups in the past 24 hours among 169 younger and older adolescents by the sex of the respondents. It was found that almost $100 \%$ of the 170 adolescents were consuming grains, white root tubers, and plantains in their diet. Older adolescents 
171 consumed dairy products and pulses slightly more (83\% and 63\% approx.) compared to younger 172 adolescents ( $80 \%$ and 58\% approx.). More than half of the adolescents ate fruits and vegetables 173 the previous day. Females were found to be eating fruits more compared to males, particularly 174 among older adolescents. Mainly, intake of older adolescents was found to be higher than younger 175 adolescents in most food groups. Only one-third of the adolescents ate dark green leafy vegetables. 176 Male adolescents consumed more nuts and seeds, especially in the group of younger adolescents 177 (around 29\%) than females (24\%). The gender differentials were quite prominent with regards to 178 the consumption of non-vegetarian foods among adolescents. Intake of eggs was almost double in 179 the case of males (18\%) compared to females $(9 \%)$ in older adolescents. A similar pattern was 180 observed in the consumption of meat, poultry, and fish in both younger and older adolescents.

181 Table 2 shows the prevalence of minimum dietary diversity where Bihar portrays better dietary 182 habits (MDD $=61 \%$ in both males and females) than UP (MDD $=58 \%$ in males and $54 \%$ in females). 183 Females faced more discrimination in UP compared to Bihar. Advancement in age leads to a 184 significant increase in MDD in the case of female adolescents in both UP and Bihar. Growing level 185 of education of the adolescents and that of mother showed a positive effect on dietary diversity of 186 the adolescents. Huge gender divide was evident among illiterate adolescents of UP, where males' 187 diet was almost twice as diverse $(\mathrm{MDD}=47 \%)$ in comparison to that of females $(\mathrm{MDD}=26 \%)$. 188 Better dietary diversity was observed in female adolescents of Bihar who had studied for 10 years 189 or more and whose mothers had studied for 8 years or more. Respondents who were working in 190 the past year consumed a less diverse diet than those not working. Adolescents belonging to SC/ST 191 caste were less likely to have a diverse diet than OBC, General, and other caste people, especially 192 in the case of UP. Adolescents from urban areas, those belonging to households with better wealth 193 status, received a more diverse diet the previous day than their counterparts. Dietary diversity was 194 found to be highly aligned with the media exposure and food consumption behavior of the 195 household, as those with low media exposure and from low FCS households had MDD as low as $19652 \%$. In contrast, those with high media exposure and high consumption households had MDD as 197 high as $75 \%$ in the case of adolescents of UP. Media exposure was positively associated with 198 minimum dietary diversity, as those with high media exposure had their minimum dietary diversity 199 as high as 74\%. Living arrangement in the household was also found to affect the intake of a varied 200 diet. Less siblings and presence of grandparents in the household was positively associated with 201 intake of a diverse and nutritious diet by the adolescent. 


\section{Results from Multivariate analysis}

203 Table 3 shows output of Binary logistic regression analysis. We find out that caste, media 204 exposure, wealth index, and household food consumption status highly influence the minimum 205 dietary diversity of adolescents. Those belonging to the OBC caste were at 1.3 times higher odds 206 of having minimum dietary diversity (A.O.R. $=1.3,95 \%$ CI (1.1-1.5)) than adolescents of SC/ST 207 caste. Wealth of the household determined the pattern of diet in the state of Bihar in such a way, 208 where adolescents from the richest category households had $90 \%$ higher likelihood of achieving 209 minimum dietary diversity than those from the poorest category households (95\% CI (1.2-3.1)). 210 Adolescents belonging to households with high food consumption were three times more likely to

211 have a diverse diet compared to those belonging to households with low food consumption 212 (A.O.R. $=3.0,95 \%$ CI (2.3-3.8) for UP; A.O.R. $=2.2,95 \%$ CI (1.6-2.9) for Bihar; A.O.R. $=2.6,95 \%$ 213 CI (2.1-3.1) overall). Adolescents highly exposed to media were twice as likely to have a minimum 214 dietary diversity compared to those with low media exposure (A.O.R.=2.1, 95\% CI (1.7-2.7)). 215 Better education level corresponded to better dietary diversity in the state of U.P. Overall, 216 adolescents from Bihar were $20 \%$ more likely to have a minimum dietary diversity than those from 217 Uttar Pradesh.

\section{Discussion}

219 In the present study, around half of the adolescents and young adults don't have an adequately 220 diverse diet. Higher gender disparities exist in UP compared to Bihar. Bihar also performs better 221 in terms of higher MDD levels. MDD is highly influenced by gender, education, caste, and media 222 exposure of the individual, while wealth index, food consumption, and caste play an essential role 223 at the household level.

224 It is observed that the most widely consumed food group among all the respondents is "grains, 225 white root tubers, and plantains". Food groups consumed by more than half of the individuals 226 include dairy, fruits, pulses, and vegetables. Similarly, a study conducted in Iran found almost all 227 the female adolescents consuming cereals, and more than half of them consuming fruits and 228 vegetables (21).

229 Gender-based discrimination is still prevalent in northern, central, and eastern zones of India (22). 230 For instance, females across India consume nutrient-rich food less frequently compared to males 
231 (23). Our study shows considerable differences in the non-vegetarian diet practices among male 232 and female adolescents in UP and Bihar. Females have consumed more fruits, whereas males 233 consumed more eggs and meat. This is observed among both age groups, but the gap is even wider 234 in the case of older adolescents. Likewise, a study based in rural India found gender disparity in 235 the dietary pattern of adolescents (24), and it corroborates with another study conducted among 236 adolescents in Bangladesh, where the inadequate dietary deficiency among adolescent girls and 237 boys was $55.5 \%$ and $50 \%$, respectively (25). Contrary to that, a study from Australia did not find 238 highly significant differences in the dietary pattern of male and female adolescents (26). Further, 239 a longitudinal study using data from Peru, Vietnam, India, and Ethiopia didn't find marked gender 240 differentials in dietary patterns of the adolescents (27).

241 Many studies from developing countries have found dietary diversity to be significantly associated 242 with the family's economic status $(21,28-31)$. Our study findings concur with the previous study 243 findings that adolescents belonging to the wealthiest quintile have higher odds of reporting MDD 244 than poorer quintiles. A study in Gujarat, India, found that higher income of the family was 245 positively associated with a higher diversity score (32). A study conducted in Bangladesh also 246 stated the positive role of Socio-economic status in MDD among adolescents (33). Lower income 247 can affect the family's purchasing power, and therefore their access and choices to various food 248 items are constrained $(34,35)$.

249 The choices of food in the initial years impact dietary patterns in later part of life also (36). Our 250 study suggests the same where adolescents with medium and high household food consumption 251 are more likely to have MDD than those with low household food consumption. This pattern is 252 observed both in UP and Bihar.

253 Mass media is popular among adolescents. Television and magazines impact adolescents' dietary 254 habits relatively more than other media both in developed and developing countries (37). Our study 255 also finds the role of mass media exposure to be positively associated with the higher dietary score 256 in the two states. Similarly, a study based in India found social media exposure to be significantly 257 associated with the high MDD among adolescent girls (24). At the same time, a study in Austria 258 found that exposure to mass media increases fruit consumption among adolescents (38).

259 The limitation of our study is that it uses a proxy measure of MDD and FCS as there was a lack of 260 complete information required to compute the standard estimate. Few adjustments are made in 
261 data so that the represented measure is as close to the standard one. A longitudinal analysis would 262 have helped establish causality of various linkages if the survey team had asked the same questions 263 at both time points. But due to a different set of questions being asked related to the dietary pattern, 264 the analysis is done only on the wave- 2 dataset, and hence the inference is from cross-sectional 265 data's point of view.

\section{Conclusion}

267 In developing countries, lack of food diversity due to constraints in access to different food groups 268 and monotonous consumption of certain cereals or food groups impedes achieving optimal 269 nutrition status. Therefore, there is a need to reemphasize the importance of dietary diversity.

270 Vegetables and fruits intake is as important as compared to that of cereals and pulses as it contain 271 a lot of fiber (39). Awareness campaigning is needed on increasing the intake of vegetables as 272 much as that of grains. The industrialization in agriculture and the focus of the government's Public 273 Distribution System (PDS) on mainly wheat and rice has reduced emphasis on many other nutrient274 rich kinds of cereal like Bajra, Ragi, Millets, etc. (40) Government can add such variety to its 275 present schemes like PDS, Mid-day meal, Antyodaya Anna Yojana, etc., and intensify awareness 276 on its importance. Our study findings indicate several factors that have a bearing on dietary 277 diversity. Foremost, being the low Socio-economic gradients and food consumption of the 278 household, which has a significant influence on the dietary patterns. Those from wealthier families 279 and higher caste have better dietary diversity. Media can be an essential tool for disseminating 280 messages and raising awareness regarding improving variety in diet among adolescents and young 281 adults. Also, the role of gender-based inequalities being responsible for the inadequate diet among 282 females can't be ruled out.

283 In the past decades, undernutrition among the under-5 has been studied extensively, but that of 284 adolescents and young adults has not received much attention. Even though the young adults (aged 28520 and above) performed better in their dietary intake, they are also far from the optimum 286 nutritional diet. Adolescence marks an important stage from childhood to adulthood. Especially 287 working on nutrition of female adolescents will result in breaking the malnutrition cycle (41). The 288 government has started taking cognizance of adolescent health issues with the launch of Rashtriya 289 Kishor Swasthya Karyakram. But, the health indicators among adolescents are still poor. Data 
290 shows that the pace of improvement is slow and not enough to attain the SDG targets by 2030. 291 India having the highest adolescent population in the world, should harness its demographic 292 dividend. And to do so, it should invest in the nutritional status and overall health of the 293 adolescents.

\section{References}

295 1. UNICEF. Adolescent Demographics - UNICEF DATA [Internet]. 2018 [cited 2021 Feb 296 13]. Available from: https://data.unicef.org/topic/adolescents/demographics/

297 2. India U. Adolescent development and participation [Internet]. [cited 2021 May 31]. 298 Available from: https://www.unicef.org/india/what-we-do/adolescent-developmentparticipation

3. Isabirye N, Bukenya JN, Nakafeero M, Ssekamatte T, Guwatudde D, Fawzi W. Dietary diversity and associated factors among adolescents in eastern Uganda: A cross-sectional study. BMC Public Health. 2020 Apr 19;20(1):534.

5. Cunningham K, Pries A, Erichsen D, Manohar S, Nielsen J. Adolescent Girls' Nutritional

6. Akseer N, Al-Gashm S, Mehta S, Mokdad A, Bhutta ZA. Global and regional trends in the nutritional status of young people: a critical and neglected age group. Annals of the New York Academy of Sciences. 2017 Apr 1;1393(1):3-20.

7. Ministry of Health and Family Welfare, Government of India, Council Population.

314 8. Dewey KG, Begum K. Long-term consequences of stunting in early life. Maternal and Child Nutrition. 2011 Oct;7(SUPPL. 3):5-18. Comprehensive National Nutrition Survey: 2016-2018. 2020.

9. Rao M, Afshin A, Singh G, Mozaffarian D. Do healthier foods and diet patterns cost more than less healthy options? A systematic review and meta-analysis. Vol. 3, BMJ Open. British Medical Journal Publishing Group; 2013. p. 4277. 
319 10. Gupta M Das. Selective discrimination against female children in rural Punjab, India. $320 \quad$ Population and development review. 1987;77-100.

321 11. Behrman JR. Intrahousehold allocation of nutrients in rural India: Are boys favored? Do 322 parents exhibit inequality aversion? Oxford Economic Papers. 1988;40(1):32-54.

323 12. Kehoe SH, Krishnaveni G V, Veena SR, Guntupalli AM, Margetts BM, Fall CHD, et al. Diet patterns are associated with demographic factors and nutritional status in S outh I ndian children. Maternal \& child nutrition. 2014;10(1):145-58.

13. Subramanian S, Deaton A. Gender effects in Indian consumption patterns. Sarvekshana. $1991 ; 14(4): 1-12$.

14. Borooah VK. Gender bias among children in India in their diet and immunisation against disease. Social Science and Medicine. 2004 May 1;58(9):1719-31.

15. Wasnik V, Rao BS, Rao DS. A Study of the Health Status of Early adolescent Girls residing in Social Welfare Hostels in Vizianagaram district of Andhra Pradesh State, India.

16. Nguyen PH, Scott S, Neupane S, Tran LM, Menon P. Social, biological, and programmatic International Journal of Collaborative Research on Internal Medicine \& Public Health. factors linking adolescent pregnancy and early childhood undernutrition: a path analysis of India's 2016 National Family and Health Survey. The Lancet Child and Adolescent Health. 2019 Jul 1;3(7):463-73.

17. Asia Lainchaur S, Marg Kathmandu L. UNICEF Regional Office for Child marriage, adolescent pregnancy and school dropout in South Asia.

340 18. Santhya KG, Acharya R, Pandey N, Gupta AK, Rampal S, Singh SK, et al. Understanding the lives of adolescents and young adults (UDAYA) in Uttar Pradesh, India. New Delhi:

343 19. Davis U. Minimum Dietary Diversity for Women- A Guide to Measurement.

344 20. Fiedler JL, Lividini K, Bermudez OI, Smitz MF. Household Consumption and Expenditures Surveys (HCES): a primer for food and nutrition analysts in low- and middle-income countries. Food and nutrition bulletin. 2012;33(3 Suppl).

21. Vakili M, Abedi P, Sharifi M, Hosseini M. Dietary diversity and its related factors among adolescents: a survey in Ahvaz-Iran. Global journal of health science. 2013;5(2):181-6. 
22. Landry M, Vyas A, Malhotra G, Nagaraj N. Adolescents' development of gender equity attitudes in India. International Journal of Adolescence and Youth. 2020;25(1):94-103.

23. Aurino E. Do boys eat better than girls in India? Longitudinal evidence on dietary diversity and food consumption disparities among children and adolescents. Economics and Human Biology. 2017 May 1;25:99-111.

24. Nithya DJ, Bhavani R V. Dietary diversity and its relationship with nutritional status among adolescents and adults in rural India. Journal of Biosocial Science. 2018 May 1;50(3):397413.

25. Akter F, Hossain MM, Shamim AA, Hasan M, Hanif AAM, Hossaine M, et al. Inadequate

26. Appannah G, Pot GK, Huang RC, Oddy WH, Beilin LJ, Mori TA, et al. Identification of a

27. Aurino E, Fernandes M, Penny ME. The nutrition transition and adolescents' diets in low-

29. Agustina R, Nadiya K, Andini EA, Setianingsih AA, Sadariskar AA, Prafiantini E, et al. and middle-income countries: A cross-cohort comparison. Public Health Nutrition. 2017 Jan 1;20(1):72-81.

28. Halala Handiso Y, Belachew T, Abuye C, Workicho A. Low dietary diversity and its determinants among adolescent girls in Southern Ethiopia. Cogent Food \& Agriculture. 2020 Jan 1;6(1):1832824.

Associations of meal patterning, dietary quality and diversity with anemia and overweightobesity among Indonesian school-going adolescent girls in West Java. Gebremedhin S, editor. PLOS ONE. 2020 Apr 23;15(4):e0231519.

30. Tamiru D, Argaw A, Gerbaba M, Nigussie A, Ayana G, Belachew T. Improving dietary diversity of school adolescents through school based nutrition education and home gardening in Jimma Zone: Quasi-experimental design. Eating Behaviors. 2016 Dec $1 ; 23: 180-6$.

31. Mersha Birru S, Tariku A, Belew AK. Improved dietary diversity of school adolescent girls in the context of urban Northwest Ethiopia: 2017. 
32. Singh S, Jones AD, DeFries RS, Jain M. The association between crop and income diversity and farmer intra-household dietary diversity in India. Food Security. 2020 Apr 1;12(2):36990.

33. Islam MR, Rahman SM, Tarafder C, Rahman MdM, Rahman A, Ekström E-C. Exploring Rural Adolescents' Dietary Diversity and Its Socioeconomic Correlates: A Cross-Sectional Study from Matlab, Bangladesh. Nutrients. 2020 Jul 26;12(8):2230.

34. Irving F. The Purchasing Power of Money: Its' Determination And Relation to Credit ... 1911.

35. Kaufman PR, Macdonald JM, Lutz SM, Smallwood DM. Do the Poor Pay More for Food? Item Selection and Price Differences Affect Low-Income Household Food Costs. 1997.

36. Birch DrL, Savage JS, Ventura A. Influences on the Development of Children's Eating

37. Nutrition in adolescence-Issues and Challenges for the Health Sector Issues in Adolescent

41. Prakash J. Why Breaking The Deadly Cycle Of Malnutrition Is Critical. 2021 Apr 30 [cited

38. Freisling H, Haas K, Elmadfa I. Mass media nutrition information sources and associations with fruit and vegetable consumption among adolescents. Public Health Nutrition. 2010;13(2):269-75.

39. Slavin JL, Lloyd B. Health Benefits of Fruits and Vegetables. Advances in Nutrition. 2012 Jul;3(4):506.

40. Programme M of S and, Programme TWF. Food and Nutrition Security Analysis, India, 2019. Ministry of Statistics and Programme Implementation \& The World Food Programme. 2019; breaking-the-cycle-of-malnutrition-is-critical/381627 
Tables

Table 1: Percentage of intake of different food groups in the past 24 hours among younger and older adolescents, Udaya study, 2018-19

\begin{tabular}{|c|c|c|c|c|c|c|}
\hline \multirow{2}{*}{ Food Groups } & \multicolumn{3}{|c|}{ Younger (12-17 years) } & \multicolumn{3}{|c|}{ Older (18-23 years) } \\
\hline & Male & Female & Significance & Male & Female & Significance \\
\hline Grains, White root tubers, Plantains & 100.0 & 99.5 & & 99.6 & 98.5 & $* * *$ \\
\hline Dairy & 80.9 & 77.8 & $* * *$ & 83.4 & 82.1 & $* * *$ \\
\hline Other and vitamin-A rich Fruits & 63.7 & 67.2 & $* * *$ & 64.2 & 71.2 & $* * *$ \\
\hline Pulses (beans, peas and lentils) & 58.6 & 58.8 & & 63.0 & 61.6 & $* * *$ \\
\hline Other and vitamin-A rich Vegetables & 56.9 & 61.7 & & 59.7 & 61.3 & \\
\hline Dark Green leafy vegetables & 33.8 & 32.5 & & 32.8 & 32.1 & \\
\hline Nuts and seeds & 28.6 & 24.1 & $* * *$ & 29.7 & 26.6 & $* * *$ \\
\hline Meat, poultry and fish & 16.5 & 13.6 & $* *$ & 17.2 & 12.4 & $* * *$ \\
\hline Eggs & 16.7 & 10.8 & $* * *$ & 17.6 & 9.2 & $* * *$ \\
\hline
\end{tabular}

Note: Significance is computed to see differences among males and females using chi-square test, where $* * *$ represents $\mathrm{p}<.01$ and $* *$ represents $\mathrm{p}<.05$

Table 2: Percentage of Minimum Dietary Diversity w.r.t. demographic and other background characteristics, Uttar Pradesh and Bihar, 2018-19

\begin{tabular}{|c|c|c|c|c|c|c|}
\hline & \multicolumn{3}{|c|}{ Males } & \multicolumn{3}{|c|}{ Females } \\
\hline & UP & Bihar & Overall & UP & Bihar & Overall \\
\hline Outcome Variable & & & $* *$ & & & $* * *$ \\
\hline Minimum Dietary Diversity & 57.9 & 61.0 & 58.8 & 53.5 & 61.1 & 56.3 \\
\hline \multicolumn{7}{|l|}{ Independent Variables } \\
\hline Age & ns & ns & ns & $* *$ & $* * *$ & $* * *$ \\
\hline $12-15$ yrs & 56.6 & 61.2 & 58.2 & 48.4 & 54.8 & 51.8 \\
\hline 16-17 yrs & 57.0 & 62.2 & 58.8 & 57.6 & 67.4 & 60.1 \\
\hline $18-20$ yrs & 57.9 & 59.5 & 58.1 & 54.8 & 67.2 & 58.1 \\
\hline $19-23$ yrs & 63.0 & 61.4 & 62.6 & 59.1 & 69.0 & 61.5 \\
\hline Completed years of education & $* * *$ & $* * *$ & $* * *$ & $* * *$ & $* * *$ & $* * *$ \\
\hline None & 46.5 & 59.5 & 50.3 & 25.8 & 50.3 & 33.0 \\
\hline $1-4$ & 55.3 & 53.5 & 54.8 & 42.2 & 47.1 & 46.1 \\
\hline 5-7 & 56.4 & 56.4 & 56.4 & 50.2 & 59.3 & 54.3 \\
\hline 8-9 & 55.5 & 66.4 & 58.8 & 51.9 & 55.6 & 53.4 \\
\hline 10-11 & 58.3 & 59.4 & 58.7 & 60.7 & 75.5 & 64.9 \\
\hline 12 and above & 63.7 & 64.4 & 63.6 & 62.1 & 74.2 & 64.4 \\
\hline Involved in paid work for the last 12 months & $* * *$ & $* * *$ & $* * *$ & $*$ & ns & $* * *$ \\
\hline Yes & 53.9 & 55.6 & 54.2 & 52.9 & 59.3 & 54.3 \\
\hline No & 60.5 & 63.4 & 61.4 & 53.7 & 61.4 & 57.0 \\
\hline Mother's Completed years of education & $* * *$ & $* * *$ & $* * *$ & $* * *$ & $* * *$ & $* * *$ \\
\hline None & 55.2 & 59.0 & 56.5 & 52.7 & 58.7 & 54.7 \\
\hline $1-7 \mathrm{yrs}$ & 55.2 & 73.1 & 60.1 & 42.5 & 61.8 & 49.3 \\
\hline 8-9yrs & 68.3 & 64.1 & 67.1 & 59.8 & 71.6 & 60.2 \\
\hline 10 and above & 66.8 & 64.3 & 66.0 & 65.2 & 73.7 & 68.5 \\
\hline
\end{tabular}




\begin{tabular}{lllllll}
\hline Caste & $* * *$ & $* * *$ & $* * *$ & $* * *$ & $* * *$ & $* * *$ \\
\hline SC/ST & 47.3 & 52.3 & 48.8 & 50.3 & 60.8 & 53.8 \\
\hline OBC & 61.8 & 62.8 & 62.1 & 53.0 & 58.8 & 55.8 \\
\hline General/Others & 62.0 & 68.3 & 63.3 & 57.7 & 73.7 & 60.2 \\
\hline Place of residence & $* * *$ & $* * *$ & $* * *$ & $* *$ & $* *$ & $* * *$ \\
\hline Urban & 61.8 & 65.3 & 62.7 & 55.1 & 70.0 & 58.9 \\
\hline Rural & 56.5 & 59.8 & 57.6 & 52.9 & 59.6 & 54.9 \\
\hline Wealth index of the household & $* * *$ & $* * *$ & $* * *$ & $* * *$ & $* * *$ & $* * *$ \\
\hline Poorest & 42.0 & 52.6 & 46.8 & 33.1 & 55.6 & 40.9 \\
\hline Poorer & 51.5 & 57.8 & 54.1 & 56.8 & 46.4 & 54.3 \\
\hline Middle & 58.7 & 57.5 & 58.3 & 57.7 & 58.7 & 58.4 \\
\hline Richer & 59.7 & 67.3 & 61.9 & 52.3 & 70.2 & 57.1 \\
\hline Richest & 65.4 & 76.1 & 67.0 & 58.7 & 79.7 & 61.9 \\
\hline Food Consumption Score index & $* * *$ & $* * *$ & $* * *$ & $* * *$ & $* * *$ & $* * *$ \\
\hline Low & 45.1 & 52.6 & 47.3 & 40.2 & 48.9 & 42.8 \\
\hline Medium & 67.1 & 61.9 & 65.5 & 57.9 & 63.8 & 58.9 \\
\hline High & 71.5 & 71.4 & 71.6 & 66.7 & 72.7 & 70.0 \\
\hline Media Exposure & $* * *$ & $* * *$ & $* * *$ & $* * *$ & $* * *$ & $* * *$ \\
\hline Low & 49.1 & 57.7 & 52.0 & 49.5 & 55.8 & 51.3 \\
\hline Medium & 58.1 & 59.0 & 58.4 & 56.3 & 68.9 & 60.9 \\
\hline High & 75.2 & 73.4 & 74.6 & 71.1 & 82.9 & 71.8 \\
\hline Number of siblings & $\mathbf{n s}$ & $*$ & $* *$ & $* * *$ & $\mathbf{n s}$ & $* * *$ \\
\hline$<\mathbf{2}$ & 60.4 & 69.3 & 62.5 & 60.5 & 66.7 & 62.2 \\
\hline 2-4 & 56.5 & 59.4 & 57.3 & 52.8 & 61.3 & 56.3 \\
\hline 4+ & 60.0 & 61.7 & 60.5 & 53.1 & 59.1 & 54.8 \\
\hline Presence of Grandparent in the household & $* * *$ & $* *$ & $* * *$ & $*$ & $\mathbf{n s}$ & $* * *$ \\
\hline No & 57.0 & 59.3 & 57.6 & 53.0 & 60.3 & 55.9 \\
\hline Yes & 61.0 & 67.4 & 63.0 & 55.2 & 64.2 & 58.1 \\
\hline Nor Sin & & & \\
\hline
\end{tabular}

421 Note: Significance is computed to see differences among males and females using chi-square test, where $* * *$

Table 3: Binary logistic regression results presenting Adjusted Odds ratio (AOR) w.r.t. various determinants predicting minimum dietary diversity among adolescents in UP and

Bihar, 2018-19

\begin{tabular}{llll}
\hline \multicolumn{1}{l}{ State } & UP & Bihar & Overall \\
\hline Uttar Pradesh & & & $1.2(1-1.4)^{* *}$ \\
\hline Bihar & & & \\
\hline Sex of respondent & & & $0.9(0.8-1.1)$ \\
\hline Male & $0.8(0.7-1)$ & $1(0.8-1.3)$ & \\
\hline Female & & & $1.2(1-1.5)$ \\
\hline Age & & & $1.0(0.8-1.2)$ \\
\hline $\mathbf{1 0 - 1 2}$ yrs & $1.1(0.8-1.5)$ & $1.3(0.9-2)$ & $1(0.7-1.4)$ \\
\hline $\mathbf{1 5 - 1 4}$ yrs & $0.9(0.7-1.3)$ & & \\
\hline
\end{tabular}




\begin{tabular}{|c|c|c|c|}
\hline 18-19 yrs & $1.1(0.8-1.6)$ & $1(0.6-1.5)$ & $1.1(0.8-1.5)$ \\
\hline \multicolumn{4}{|c|}{ Completed years of education } \\
\hline \multicolumn{4}{|c|}{ None } \\
\hline $1-4$ & $1.8(0.9-3.6)$ & $0.9(0.4-2.2)$ & $1.3(0.8-2.4)$ \\
\hline 5-7 & $2.1(1.2-3.7)^{* * *}$ & $1(0.5-2.1)$ & $1.5(1-2.4)$ \\
\hline 8-9 & $2.0(1.1-3.5)^{* *}$ & $1.1(0.5-2.3)$ & $1.5(1-2.3)$ \\
\hline 10-11 & $2.2(1.2-3.8)^{* * *}$ & $1(0.5-2.2)$ & $1.5(1-2.4)$ \\
\hline 12 and above & $2.2(1.2-3.8)^{* * *}$ & $1.2(0.6-2.6)$ & $1.7(1.1-2.6)^{* *}$ \\
\hline \multicolumn{4}{|c|}{ Whether involved in paid work for the last 12 months } \\
\hline \multicolumn{4}{|c|}{ Yes } \\
\hline No & $1.1(0.9-1.4)$ & $1.2(0.9-1.6)$ & $1.1(1-1.3)$ \\
\hline \multicolumn{4}{|c|}{ Mother's Completed years of education } \\
\hline \multicolumn{4}{|c|}{ None } \\
\hline $1-7$ yrs & $0.8(0.6-1)$ & $1.3(0.8-1.9)$ & $0.9(0.7-1.2)$ \\
\hline 8-9yrs & $1.3(0.9-1.8)$ & $1(0.6-1.6)$ & $1.2(0.9-1.6)$ \\
\hline 10and above & $1.2(0.9-1.7)$ & $0.7(0.5-1.1)$ & $1.1(0.8-1.4)$ \\
\hline \multicolumn{4}{|l|}{ Caste } \\
\hline \multicolumn{4}{|l|}{ SC/ST } \\
\hline OBC & $1.4(1.1-1.7)^{* * *}$ & $1.1(0.8-1.4)$ & $1.3(1.1-1.5)^{* *}$ \\
\hline General/Others & $1.3(1-1.7)$ & $1.3(0.8-2)$ & $1.3(1-1.6)$ \\
\hline \multicolumn{4}{|l|}{ Place of residence } \\
\hline \multicolumn{4}{|l|}{ Urban } \\
\hline Rural & $1.2(0.9-1.4)$ & $1(0.8-1.3)$ & $1.1(0.9-1.3)$ \\
\hline \multicolumn{4}{|c|}{ Wealth index of the household } \\
\hline \multicolumn{4}{|c|}{ Poorest } \\
\hline Poorer & $1.5(1-2.1)$ & $0.9(0.6-1.4)$ & $1.2(0.9-1.5)$ \\
\hline Middle & $1.7(1.2-2.4)^{* * *}$ & $1.0(0.7-1.5)$ & $1.3(1-1.7)^{* *}$ \\
\hline Richer & $1.5(1-2.1)^{* *}$ & $1.4(0.9-2.2)$ & $1.4(1-1.8)^{* *}$ \\
\hline Richest & $1.4(0.9-2)$ & $1.9(1.2-3.1)^{* *}$ & $1.3(1-1.9)$ \\
\hline \multicolumn{4}{|c|}{ Food Consumption Score index } \\
\hline \multicolumn{4}{|c|}{ Low } \\
\hline Medium & $2.0(1.6-2.5)^{* * *}$ & $1.4(1-1.9)^{* *}$ & $1.8(1.5-2.1)^{* * *}$ \\
\hline High & $3.0(2.3-3.8)^{* * *}$ & $2.2(1.6-2.9)^{* * *}$ & $2.6(2.1-3.1)^{* * *}$ \\
\hline \multicolumn{4}{|l|}{ Media Exposure } \\
\hline \multicolumn{4}{|l|}{ Low } \\
\hline Medium & $1.3(1-1.6)^{* *}$ & $1.1(0.8-1.4)$ & $1.2(1-1.4)^{* *}$ \\
\hline High & $2.3(1.7-3.1)^{* * *}$ & $1.8(1.2-2.6)^{* * *}$ & $2.1(1.6-2.7)^{* * *}$ \\
\hline \multicolumn{4}{|c|}{ Number of siblings } \\
\hline \multicolumn{4}{|c|}{$<2$} \\
\hline $2-4$ & $1.0(0.7-1.3)$ & $0.8(0.5-1.3)$ & $0.9(0.7-1.2)$ \\
\hline $4+$ & $1.2(0.9-1.7)$ & $0.8(0.5-1.3)$ & $1.1(0.8-1.4)$ \\
\hline \multicolumn{4}{|c|}{ Presence of Grandparent in the household } \\
\hline \multicolumn{4}{|c|}{ No } \\
\hline Yes & $1.0(0.8-1.3)$ & $1.2(0.9-1.5)$ & $1.1(0.9-1.3)$ \\
\hline
\end{tabular}

